\title{
ON THE POSSIBLE ANALOGY BETWEEN SPIN-GLASSES AND SYSTEMS CONTAINING DX CENTERS
}

\author{
A. EUSAKOWSKI \\ Institute of Physics, Polish Academy of Sciences \\ Al. Lotników 32/46, 02-668 Warszawa, Poland
}

\begin{abstract}
An analogy between a spin-glass and a material containing DX centers is postulated. The basis of the analogy is the formal identity of the Hamiltonians of these two systems. A correspondence between parameters of the spin-glass and of the DX system is established. Experiments which could support the above idea are proposed.
\end{abstract}

PACS numbers: $75.10 . \mathrm{Nr}, 71.55 . \mathrm{Dp}$

During last years number of papers have been devoted to the problem of correlations between charged defects in semiconductors. One of the most cxtensively studied material in which this phenomenon is observed is IIgSe doped with Fe. Recently, it became clear that these correlations should also be taken into account in systems containing deep impurities, the so-called DX centers $[1,2]$. In the present contribution I would like to show that there is a certain similarity between descriptions of a system of DX centers and a spin-glass.

Let us consider $N$ DX centers placed randomly in the host crystal. In the following I assume the $\mathrm{DX}^{-}$model, i.e. a $\mathrm{DX}$ center may exist only in two charge states, namely \pm 1 . Thus, the state of $i$-th DX center $(i=1, \ldots, N)$ may be described by the Ising spin variable $S_{i}$ which may be equal to +1 or -1 , depending on number of electrons ( 0 or 2$)$ localized on the center.

The Coulomb energy of the system is equal to

$$
E_{\mathrm{coul}}=\sum_{i \neq j} J_{i j} S_{i} S_{j}
$$

where

$$
J_{i j}=\frac{e^{2} \mathrm{e}^{-\kappa \mid} \overline{\boldsymbol{r}}_{i}-\bar{r}_{j} \mid}{\varepsilon\left|\bar{r}_{i}-\bar{r}_{j}\right|}
$$

is the screened Coulomb potential energy, $\bar{r}_{i}, \bar{r}_{j}$ are positions of $i$-th and $j$-th centers and $\kappa$ is the inverse screening radius.

Formally, $E_{\text {coul }}$ has the same form as the exchange energy in magnetic systems with antiferromagnetic interactions between spins. Because of randomness 
in positions of impurities one may suspect frustration and consequently a very complicated distribution of energy levels in the configurational space.

Now $n, N_{+}, N_{-}$are the number of electrons in the conduction band and numbers of $\mathrm{DX}^{+}$and $\mathrm{DX}^{-}$centers, respectively. The crystal neutrality condition $n=N_{+}-N_{-}$may be written in the form

$$
n=\sum_{i} S_{i} \text {. }
$$

Thus, the electron density in the conduction band plays the role of magnetization density in spin-glasses.

Now let us find an analog of the magnetic field. To this aim let us consider a DX center occupied by two electrons. Let $2 H$ be the energy required to transfer these two electrons into the conduction band. This energy is proportional to the difference between the DX level and the Fermi energy of electrons in the conduction band. If $\delta n$ electrons are transfered, the change of the system's energy is equal to $H \delta n$, so $H$ corresponds to the magnetic field. However, this analogy is not complete because $H$ is $n$ dependent due to $n$ dependence of the Fermi energy.

The relative position of the DX level and the bottom of the conduction band changes when the hydrostatic pressure is applied, so it is possible to measure the quantity $(\delta n / \delta p)$ which is the analog of magnetic susceptibility. It turns out that in some approximation this "susceptibility" may be obtained from measurements of the mobility. The argument goes as follows.

The enhancement $\Delta \mu$ of the mobility caused by a spatial ordering of the charged scaterers may be described with the aid of the connected "spin"-"spin" correlation function $C\left(\bar{r}_{i}-\bar{r}_{j}\right)=\left\langle S_{i} S_{j}\right\rangle-\left\langle S_{i}\right\rangle\left\langle S_{j}\right\rangle$ which enters the formula for $\Delta \mu$ via its Fourier transform $C(\bar{q})[1]$ :

$$
\Delta \mu \propto \int_{0}^{2 k} \mathrm{~d} q|U(q)|^{2} q^{3} C(q),
$$

where $k$ is the module of the Fermi wave vector and $U(\bar{q})$ is the Fourier transform of the screened Coulomb potential. We suspect that relevant changes of $C(\bar{q})$ occur for $q$ in the vicinity of $a^{-1}$, where $a$ is the average distance between impurities. If the Fermi wavelength is much larger than $a$, one may approximate $C(\bar{q}) \approx C(0)$ for $0<q<2 k$ and, consequently, $\Delta \mu$ becomes proportional to $C(0)$. Since in magnetic systems $C(0)$ is proportional to the susceptibility $\chi$ one finds a correspondence between $\Delta \mu$ and $\chi$.

Let us consider now shortly a possible description of the system's dynamics. The transition of electrons from one DX center to another takes place via the conduction band. Since in such a process, locally, the charge ("spin") is not conserved it seems plausible that the dynamics of Glauber type, widely used in dynamical description of spin-glasses should be also applicable in the present case. One should remember, however, that the time of "spin" flipping is much longer here and it is strongly temperature dependent due to the high value of the configurational energy barier.

From the above we see that up to certain limitations, mathematical description of the system of charged impurities is closely related to that for the Ising spin-glasses. The question arises whether in a properly chosen experiment 
performed on a sample containing DX centers one may observe a behavior characteristic for spin-glasses, in particular an analog of the paramagnet-spin-glass transition.

Before postulating any experiment let us analyse whether the observation of such transition is possible at all. The main obstacle may be the already mentioned high value of configurational energy barier. About a certain temperature $T_{0}$ (usually about $100 \mathrm{~K}$ ), which depends on a material, all centers become thermally frozen. Consequently, if the transition is to be observable, its temperature $T_{\mathrm{f}}$ should be higher than $T_{0}$. In the case of magnetic systems $k_{\mathrm{B}} T_{\mathrm{f}}$ and the exchange constant are of the same order of magnitude. It turns out that the average $J_{i j}$ (see Eq. (2)) and $k_{\mathrm{B}} T_{0}$ are of the same order, also. Thus it seems that the observation of the transition, at least in principle, is not excluded.

Consider now mobility measurements. We observe more or less sharp increase of the mobility at temperatures which are close to $T_{0}$ [2]. This proves that the process of ordering of charged donors takes place and the correlations effects are really important even at relatively high temperatures.

Let me propose now two experiments.

First of all, it would be very interesting to compare experimental temperature dependence of $\Delta \mu$ and $(\delta n / \delta p)$. Such comparison would provide the test of the present approach.

The second proposition is to measure the time dependence of $\Delta \mu$ at different temperatures. One can introduce disorder into the lattice of DX centers by, for example, illumination of the sample. The observation of the relaxation of $\Delta \mu$ to the equilibrium value would give us some information about the dynamics of the lattice of charged donors. The change of the behavior of $\Delta \mu(t)$ (for example from exponential to algebraic dependence) at certain temperature higher than $T_{0}$ would be a hint that the transition really takes place.

\section{References}

[1] J. Kossut, Z. Wilamowski, T. Dietl, K. Świątek, Acta Phys. Pol. A79, 49 (1991).

[2] Z. Wilamowski, W. Jantsch, G. Ostermayer, J. Kossut, Material Science Forum 83-87, 805 (1992). 